\title{
On the Kinematics for the Closed Planar Homothetic Inverse Motions in Complex Plane
}

\author{
Onder Sener ${ }^{1}$, Ayhan Tutar ${ }^{1,2 *}$ \\ ${ }^{1}$ Faculty of Art and Science, Ondokuz Mayis University, Samsun, Turkey \\ ${ }^{2}$ Department of Mathematics, Faculty of Science, Kyrgyz-Türk Manas University, Bishkek, Kyrgyzstan \\ Email: *atutar@omu.edu.tr
}

How to cite this paper: Sener, O. and Tutar, A. (2017) On the Kinematics for the Closed Planar Homothetic Inverse Motions in Complex Plane. Journal of Applied Mathematics and Physics, 5, 1120-1129. https://doi.org/10.4236/jamp.2017.55099

Received: March 23, 2017

Accepted: May 23, 2017

Published: May 27, 2017

Copyright (c) 2017 by authors and Scientific Research Publishing Inc. This work is licensed under the Creative Commons Attribution International License (CC BY 4.0).

http://creativecommons.org/licenses/by/4.0/

\section{c) (i) Open Access}

\begin{abstract}
In this paper, the Steiner area formula and the polar moment of inertia were expressed during one-parameter closed planar homothetic inverse motions in complex plane. The Steiner point was defined when the rotation number was different zero and it was called the Steiner normal when the rotation number was equal to zero. The fixed pole point was given with its components and its relation between Steiner point or Steiner normal was explained. The sagittal motion of a telescopic crane was considered as an example. This motion was described by a double hinge consisting of the fixed control panel of the telescopic crane and the moving arm of the telescopic crane. The theoretical concepts and results were applied for this motion.
\end{abstract}

\section{Keywords}

Steiner Formula, Polar Moment of Inertia, Planar Kinematics, Homothetic Inverse Motions, Complex Plane

\section{Introduction}

For a geometrical object rolling on a line and making a complete turn, some properties of the area of a path of a point were given by Steiner [1]. The Steiner area formula and the Holditch theorem during one-parameter closed planar homothetic motions were expressed by Tutar [2]. We want to explain the relation between the Steiner formula and the polar moment of inertia for the closed planar homothetic inverse motions in complex plane and find experimental data equivalents of the theoretical results in this study. We calculated the expression of the Steiner formula relative to the fixed coordinate system under one parameter closed planar motions in complex plane. If the points of the fixed plane which enclose the same area lie on a circle, then the centre of this circle is called the Steiner point $(h=1)$ [3] [4]. If these points lie on a line, we use Steiner nor- 
mal instead of Steiner point. Then we obtained the fixed pole point for the closed planar homothetic inverse motions. We dealt with the polar moment of inertia of a path generated by a closed planar homothetic inverse motion. Moreover, we expressed the relation between the area enclosed by a path and the polar moment of inertia. As an example, the sagittal motion of a telescopic crane which is described by a double hinge being fixed and moving was considered. The Steiner area formula, the fixed pole point and the polar moment of inertia were calculated for this motion. Furthermore, the relation between the Steiner formula and the polar moment of inertia was expressed.

\section{Closed Homothetic Inverse Motions in Complex Plane}

We consider one parameter closed planar homothetic motion between two reference systems: the fixed $E^{\prime}$ and the moving $E$, with their origins $\left(O, O^{\prime}\right)$ and orientations. Then, we take into account motion relative to the moving coordinate system (inverse motion). We know the motion defined by the transformation $X^{\prime}(t)=h(t) X \mathrm{e}^{\mathrm{i} \alpha(t)}+U^{\prime}(t)$ is called one-parameter closed planar homothetic direct motion in complex plane.

By taking displacement vector $O O^{\prime}=U$ and $O^{\prime} O=U^{\prime}$, the total angle of rotation $\alpha(t)$, the motion defined by the transformation

$$
X(t)=\frac{1}{h(t)}\left(X^{\prime}-U^{\prime}(t)\right) \mathrm{e}^{-i \alpha(t)}
$$

is called one-parameter closed planar homothetic inverse motion in complex plane and denoted by $E^{\prime} / E$, where $h$ is a homothetic scale of the motion $E^{\prime} / E, X$ and $X^{\prime}$ are the position vectors with respect to the moving and fixed rectangular coordinate systems of a point $X \in E$, respectively. The homothetic scale $h$ and the vector $X$ and $U, U^{\prime}$ are continuously differentiable functions of a real parameter $t$.

In Equation (1), $X(t)$ is the trajectory with the respect to the moving system of a point $X^{\prime}$ belonging to the fixed system.

If we consider the coordinates of the equation above,

$$
\begin{gathered}
X(t)=x_{1}(t)+i x_{2}(t), \quad X^{\prime}=x_{1}^{\prime}+i x_{2}^{\prime}, \\
U^{\prime}(t)=u_{1}^{\prime}(t)+i u_{2}^{\prime}(t), \quad U(t)=u_{1}(t)+i u_{2}(t)
\end{gathered}
$$

we can write

$$
x_{1}(t)+i x_{2}(t)=\frac{1}{h(t)}\left[\left(x_{1}^{\prime}-u_{1}^{\prime}(t)\right)+i\left(x_{2}-u_{2}^{\prime}(t)\right)\right](\cos \alpha(t)-i \sin \alpha(t))
$$

From Equation (2), the components of $X(t)$ may be given as

$$
\left.\begin{array}{l}
x_{1}(t)=\frac{1}{h(t)}\left[\cos (\alpha(t))\left(x_{1}^{\prime}-u_{1}^{\prime}(t)\right)+\sin (\alpha(t))\left(x_{2}^{\prime}-u_{2}^{\prime}(t)\right)\right] \\
x_{2}(t)=\frac{1}{h(t)}\left[-\sin (\alpha(t))\left(x_{1}^{\prime}-u_{1}^{\prime}(t)\right)+\cos (\alpha(t))\left(x_{2}^{\prime}-u_{2}^{\prime}(t)\right)\right]
\end{array}\right\}
$$

If we show the coordinates of the Equation (1) as 


$$
X(t)=\left(\begin{array}{l}
x_{1}(t) \\
x_{2}(t)
\end{array}\right), \quad X^{\prime}=\left(\begin{array}{l}
x_{1}^{\prime} \\
x_{2}^{\prime}
\end{array}\right), \quad U^{\prime}(t)=\left(\begin{array}{l}
u_{1}^{\prime}(t) \\
u_{2}^{\prime}(t)
\end{array}\right), \quad U(t)=\left(\begin{array}{l}
u_{1}(t) \\
u_{2}(t)
\end{array}\right)
$$

and rotation matrix

$$
R(t)=\left(\begin{array}{cc}
\cos (\alpha(t)) & -\sin (\alpha(t)) \\
\sin (\alpha(t)) & \cos (\alpha(t))
\end{array}\right)
$$

we can obtain

$$
X(t)=\frac{1}{h(t)}(R(t))^{\mathrm{T}}\left(X^{\prime}-U^{\prime}(t)\right)
$$

If Equation (3) differentiated, we have

$$
\left.\begin{array}{rl}
\mathrm{d} x_{1}= & -\frac{\mathrm{d} h}{h^{2}}\left[\cos \alpha\left(x_{1}^{\prime}-u_{1}^{\prime}\right)+\sin \alpha\left(x_{2}^{\prime}-u_{2}^{\prime}\right)\right] \\
& +\frac{1}{h}\left[-(\cos \alpha) \mathrm{d} u_{1}^{\prime}-\left(x_{1}^{\prime}-u_{1}^{\prime}\right)(\sin \alpha) \mathrm{d} \alpha-(\sin \alpha) \mathrm{d} u_{2}^{\prime}+\left(x_{2}^{\prime}-u_{2}^{\prime}\right)(\cos \alpha) \mathrm{d} \alpha\right] \\
\mathrm{d} x_{2}= & -\frac{\mathrm{d} h}{h^{2}}\left[-\sin \alpha\left(x_{1}^{\prime}-u_{1}^{\prime}\right)+\cos \alpha\left(x_{2}^{\prime}-u_{2}^{\prime}\right)\right] \\
& +\frac{1}{h}\left[(\sin \alpha) \mathrm{d} u_{1}^{\prime}-\left(x_{1}^{\prime}-u_{1}^{\prime}\right)(\cos \alpha) \mathrm{d} \alpha-(\cos \alpha) \mathrm{d} u_{2}^{\prime}-\left(x_{2}^{\prime}-u_{2}^{\prime}\right)(\sin \alpha) \mathrm{d} \alpha\right]
\end{array}\right\}
$$

\subsection{The Steiner Formula for the Homothetic Inverse Motions}

The formula for the area $F^{\prime}$ of a closed planar curve of the point $X$ is given by

$$
F^{\prime}=\frac{1}{2} \oint\left(x_{1} \mathrm{~d} x_{2}-x_{2} \mathrm{~d} x_{1}\right) .
$$

If Equations (3) and (6) are replaced in Equation (7),

$$
\begin{aligned}
2 F^{\prime}= & -\left(x_{1}^{\prime 2}+x_{2}^{\prime 2}\right) \oint \frac{1}{h^{2}} \mathrm{~d} \alpha+x_{1}^{\prime} \oint\left(2 \frac{1}{h^{2}} u_{1}^{\prime} \mathrm{d} \alpha-\frac{1}{h^{2}} \mathrm{~d} u_{2}^{\prime}\right)+x_{2}^{\prime} \oint\left(2 \frac{1}{h^{2}} u_{2}^{\prime} \mathrm{d} \alpha+\frac{1}{h^{2}} \mathrm{~d} u_{1}^{\prime}\right) \\
& -\oint\left\{\frac{1}{h^{2}}\left(u_{1}^{\prime 2}+u_{2}^{\prime 2}\right) \mathrm{d} \alpha-\frac{1}{h^{2}}\left(u_{1}^{\prime} \mathrm{d} u_{2}^{\prime}-u_{2}^{\prime} \mathrm{d} u_{1}^{\prime}\right)\right\}
\end{aligned}
$$

is obtained. The integral coefficients in Equation (8) can be shown as

$$
\left.\begin{array}{l}
\oint\left(2 \frac{1}{h^{2}} u_{1}^{\prime} \mathrm{d} \alpha-\frac{1}{h^{2}} \mathrm{~d} u_{2}^{\prime}\right)=a^{\prime *} \\
\oint\left(2 \frac{1}{h^{2}} u_{2}^{\prime} \mathrm{d} \alpha+\frac{1}{h^{2}} \mathrm{~d} u_{1}^{\prime}\right)=b^{\prime *} \\
-\oint\left\{\frac{1}{h^{2}}\left(u_{1}^{\prime 2}+u_{2}^{\prime 2}\right) \mathrm{d} \alpha-\frac{1}{h^{2}}\left(u_{1}^{\prime} \mathrm{d} u_{2}^{\prime}-u_{2}^{\prime} \mathrm{d} u_{1}^{\prime}\right)\right\}=c^{\prime}
\end{array}\right\}
$$

If we show the trajectory of the orjin of the fixed system by $F_{o^{\prime}}^{\prime}=F^{\prime}\left(x_{1}^{\prime}=0, x_{2}^{\prime}=0\right)$, we can say

$$
2 F_{o^{\prime}}^{\prime}=c^{\prime} .
$$

The coefficient $m^{\prime}$ is defined by

$$
m^{\prime}=\oint \frac{1}{h^{2}} \mathrm{~d} \alpha=\frac{1}{h^{2}\left(t_{0}\right)} \oint \mathrm{d} \alpha=\frac{1}{h^{2}\left(t_{0}\right)} 2 \pi v
$$


with the rotation number $v$ establishes whether the lines with $F^{\prime}=$ const. describing circles or straight lines. If $v \neq 0$, then we have circles. If $v=0$, the circles reduce to straight lines. If Equations (9), (10) and (11) are substituted in Equation (8), then

$$
2\left(F^{\prime}-F_{o^{\prime}}^{\prime}\right)=-\left(x_{1}^{\prime 2}+x_{2}^{\prime 2}\right) m^{\prime}+a^{\prime *} x_{1}^{\prime}+b^{\prime *} x_{2}^{\prime}
$$

can be written.

A Different Parametrization for the Integral Coefficients Equation (5) by differentiation with respect to $t$ yields

$$
\mathrm{d} X=\frac{1}{h} \mathrm{~d}\left(R^{\mathrm{T}}\right)\left(X^{\prime}-U^{\prime}\right)-\frac{1}{h} R^{\mathrm{T}} \mathrm{d} U^{\prime}-\frac{\mathrm{d} h}{h^{2}} R^{\mathrm{T}}\left(X^{\prime}-U^{\prime}\right)
$$

and if we use $X^{\prime}=P^{\prime}=\left(\begin{array}{c}p_{1}^{\prime} \\ p_{2}^{\prime}\end{array}\right)$ for the pole point, we can write

$$
0=\mathrm{d} X=\frac{1}{h} \mathrm{~d}\left(R^{\mathrm{T}}\right)\left(P^{\prime}-U^{\prime}\right)-\frac{1}{h} R^{\mathrm{T}} \mathrm{d} U^{\prime}-\frac{\mathrm{d} h}{h^{2}} R^{\mathrm{T}}\left(P^{\prime}-U^{\prime}\right)
$$

Then if $U^{\prime}=\left(\begin{array}{l}u_{1}^{\prime} \\ u_{2}^{\prime}\end{array}\right)$ is solved from Equation (13), we find

$$
\left.\begin{array}{l}
u_{1}^{\prime}=p_{1}^{\prime}+\frac{h}{h^{2}(\mathrm{~d} \alpha)^{2}+(\mathrm{d} h)^{2}}\left(\mathrm{~d} h \mathrm{~d} u_{1}^{\prime}+h \mathrm{~d} \alpha \mathrm{d} u_{2}^{\prime}\right) \\
u_{2}^{\prime}=p_{2}^{\prime}+\frac{h}{h^{2}(\mathrm{~d} \alpha)^{2}+(\mathrm{d} h)^{2}}\left(\mathrm{~d} h \mathrm{~d} u_{2}^{\prime}-h \mathrm{~d} \alpha \mathrm{d} u_{1}^{\prime}\right)
\end{array}\right\}
$$

If Equation (14) is replaced in Equation (9),

$$
\left.\begin{array}{l}
a^{\prime *}=2 \oint \frac{1}{h^{2}} p_{1}^{\prime} \mathrm{d} \alpha+2 \oint \frac{\mathrm{d} \alpha}{h^{3}(\mathrm{~d} \alpha)^{2}+h(\mathrm{~d} h)^{2}}\left(\mathrm{~d} h \mathrm{~d} u_{1}^{\prime}+h \mathrm{~d} \alpha \mathrm{d} u_{2}^{\prime}\right)-\oint \frac{1}{h^{2}} \mathrm{~d} u_{2}^{\prime} \\
b^{\prime *}=2 \oint \frac{1}{h^{2}} p_{2}^{\prime} \mathrm{d} \alpha+2 \oint \frac{\mathrm{d} \alpha}{h^{3}(\mathrm{~d} \alpha)^{2}+h(\mathrm{~d} h)^{2}}\left(\mathrm{~d} h \mathrm{~d} u_{2}^{\prime}-h \mathrm{~d} \alpha \mathrm{d} u_{1}^{\prime}\right)+\oint \frac{1}{h^{2}} \mathrm{~d} u_{1}^{\prime}
\end{array}\right\}
$$

can be rewritten. Also Equation (15) can be expressed as separately

$$
\begin{gathered}
a^{\prime}:=2 \oint \frac{1}{h^{2}} p_{1}^{\prime} \mathrm{d} \alpha, \quad b^{\prime}:=2 \oint \frac{1}{h^{2}} p_{2}^{\prime} \mathrm{d} \alpha \\
u_{1}^{\prime}:=2 \oint \frac{\mathrm{d} \alpha}{h^{3}(\mathrm{~d} \alpha)^{2}+h(\mathrm{~d} h)^{2}}\left(\mathrm{~d} h \mathrm{~d} u_{1}^{\prime}+h \mathrm{~d} \alpha \mathrm{d} u_{2}^{\prime}\right)-\oint \frac{1}{h^{2}} \mathrm{~d} u_{2}^{\prime}, \\
u_{2}^{\prime}:=2 \oint \frac{\mathrm{d} \alpha}{h^{3}(\mathrm{~d} \alpha)^{2}+h(\mathrm{~d} h)^{2}}\left(\mathrm{~d} h \mathrm{~d} u_{2}^{\prime}-h \mathrm{~d} \alpha \mathrm{d} u_{1}^{\prime}\right)+\oint \frac{1}{h^{2}} \mathrm{~d} u_{1}^{\prime}, \quad \mu^{\prime}=\left(\begin{array}{c}
u_{1}^{\prime} \\
u_{2}^{\prime}
\end{array}\right)
\end{gathered}
$$

Using Equations (16) and (17), we have

$$
2\left(F^{\prime}-F_{o^{\prime}}^{\prime}\right)=-\left(x_{1}^{\prime 2}+x_{2}^{\prime 2}\right) m^{\prime}+a^{\prime} x_{1}^{\prime}+b^{\prime} x_{2}^{\prime}+\mu_{1}^{\prime} x_{1}^{\prime}+\mu_{2}^{\prime} x_{2}^{\prime} .
$$

\subsection{Steiner Point or Steiner Normal for the Homothetic Inverse Motions}

By taking $m^{\prime} \neq 0$, the Steiner point $S^{\prime}=\left(s_{1}^{\prime}, s_{2}^{\prime}\right)$ for the closed planar homothetic inverse motion can be written as 


$$
s_{j}^{\prime}=\frac{\oint \frac{1}{h^{2}} p_{j}^{\prime} \mathrm{d} \alpha}{\oint \frac{1}{h^{2}} \mathrm{~d} \alpha}, \quad j=1,2
$$

Then

$$
\oint \frac{1}{h^{2}} p_{1}^{\prime} \mathrm{d} \alpha=s_{1}^{\prime} m^{\prime}, \quad \oint \frac{1}{h^{2}} p_{2}^{\prime} \mathrm{d} \alpha=s_{2}^{\prime} m^{\prime}
$$

is found. If we consider Equation (19) and Equation (20) is replaced in Equation (17),

$$
2\left(F^{\prime}-F_{o^{\prime}}^{\prime}\right)=-m^{\prime}\left(x_{1}^{\prime 2}+x_{2}^{\prime 2}-2 s_{1}^{\prime} x_{1}^{\prime}-2 s_{2}^{\prime} x_{2}^{\prime}\right)+\mu_{1}^{\prime} x_{1}^{\prime}+\mu_{2}^{\prime} x_{2}^{\prime}
$$

is found. Equation (21) is called the Steiner area formula for the closed planar homothetic inverse motion.

By dividing Equation (21) by $\mathrm{m}^{\prime}$ and by completing the squares, one obtains the equation of a circle,

$$
\begin{aligned}
& \left(x_{1}^{\prime}-\left(s_{1}^{\prime}-\frac{\mu_{1}^{\prime}}{2 m^{\prime}}\right)\right)^{2}+\left(x_{2}^{\prime}-\left(s_{2}^{\prime}-\frac{\mu_{2}^{\prime}}{2 m^{\prime}}\right)\right)^{2}-\left(s_{1}^{\prime}-\frac{\mu_{1}^{\prime}}{2 m^{\prime}}\right)^{2}-\left(s_{2}^{\prime}-\frac{\mu_{2}^{\prime}}{2 m^{\prime}}\right)^{2} \\
& =-\frac{2\left(F^{\prime}-F_{o^{\prime}}^{\prime}\right)}{m^{\prime}}
\end{aligned}
$$

All the moving points of the fixed plane which pass around equal orbit areas under the motion $E / E^{\prime}$ lie on the same circle with the center

$$
M^{\prime}=\left(s_{1}^{\prime}-\frac{\mu_{1}^{\prime}}{2 m^{\prime}}, s_{2}^{\prime}-\frac{\mu_{2}^{\prime}}{2 m^{\prime}}\right)
$$

in the fixed plane.

In the case of $h(t)=1$, since $\mu_{1}^{\prime}=\mu_{2}^{\prime}=0$, the point $M^{\prime}$ and the Steiner point $S^{\prime}$ coincide [3]. Also by taking $m^{\prime}=0$, if it is replaced in Equation (18), then we have

$$
\left(a^{\prime}+\mu_{1}^{\prime}\right) x_{1}^{\prime}+\left(b^{\prime}+\mu_{2}^{\prime}\right) x_{2}^{\prime}-2\left(F^{\prime}-F_{o^{\prime}}^{\prime}\right)=0
$$

Equation (24) is a straight line. If no complete loop occurs, then $v=0$ and the circles are reduced to straight lines, in other words, to a circle whose center lies at infinity. The normal to the lines of equal areas in Equation (24) is given by

$$
n^{\prime}=\left(\begin{array}{l}
a^{\prime}+\mu_{1}^{\prime} \\
b^{\prime}+\mu_{2}^{\prime}
\end{array}\right)
$$

which is called the Steiner normal [5].

\subsection{The Fixed Pole Point for the Homothetic Inverse Motions}

Using Equation (14), if $P^{\prime}=\left(\begin{array}{l}p_{1}^{\prime} \\ p_{2}^{\prime}\end{array}\right)$ is solved,

$$
\left.\begin{array}{l}
p_{1}^{\prime}=\frac{h}{h^{2}(\mathrm{~d} \alpha)^{2}+(\mathrm{d} h)^{2}}\left(-\mathrm{d} h \mathrm{~d} u_{1}^{\prime}-h \mathrm{~d} \alpha \mathrm{d} u_{2}^{\prime}\right)+u_{1}^{\prime} \\
p_{2}^{\prime}=\frac{h}{h^{2}(\mathrm{~d} \alpha)^{2}+(\mathrm{d} h)^{2}}\left(-\mathrm{d} h \mathrm{~d} u_{2}^{\prime}+h \mathrm{~d} \alpha \mathrm{d} u_{1}^{\prime}\right)+u_{2}^{\prime}
\end{array}\right\}
$$


is found for the fixed pole point $P^{\prime}$.

For $m^{\prime} \neq 0$, using Equations (11) and (19), we arrive at the relation in Equation (20) between the Steiner point and the pole point.

For $m^{\prime}=0$, using Equations (16), (17) and (25), we arrive at the relation between the Steiner normal and the pole point below:

$$
\left(\begin{array}{l}
a^{\prime} \\
b^{\prime}
\end{array}\right)=\left(\begin{array}{l}
2 \oint \frac{1}{h^{2}} p_{1}^{\prime} \mathrm{d} \alpha \\
2 \oint \frac{1}{h^{2}} p_{2}^{\prime} \mathrm{d} \alpha
\end{array}\right)=n^{\prime}-\mu^{\prime}
$$

\subsection{The Polar Moments of Inertia for the Homothetic Inverse Motions}

Because of their mathematical structure, these averages may be interpreted as polar moments of inertia $T^{\prime}$ for a path with closed homothetic motion. In this section we want to derive a formula combining $T^{\prime}, m^{\prime}, n^{\prime}$ and the area $F^{\prime}$ together.

A relation between the Steiner formula and the polar moment of inertia around the instantaneous pole can be found in [6]. Müller [3] proved a relation to the polar moment of inertia around the origin. Tölke [7] investigated the same for closed equiaffine mappings. Düldül and Kuruoğlu [8] generalized Müller's results for homothetic motion.

If we use $\alpha$ as parametrization, we need to calculate

$$
T^{\prime}=\oint\left(x_{1}^{2}+x_{2}^{2}\right) \mathrm{d} \alpha
$$

along the path of $X$. Then, using Equation (3)

$T^{\prime}=\left(x_{1}^{\prime 2}+x_{2}^{\prime 2}\right) m^{\prime}+x_{1}^{\prime} \oint\left(-2 \frac{1}{h^{2}} u_{1}^{\prime} \mathrm{d} \alpha\right)+x_{2}^{\prime} \oint\left(-2 \frac{1}{h^{2}} u_{2}^{\prime} \mathrm{d} \alpha\right)+\oint \frac{1}{h^{2}}\left(u_{1}^{\prime 2}+u_{2}^{\prime 2}\right) \mathrm{d} \alpha$

is obtained.

We need calculate to the polar moments of inertia of the origin of the fixed system, therefore $T_{o^{\prime}}^{\prime}=T^{\prime}\left(x_{1}^{\prime}=0, x_{2}^{\prime}=0\right)$, one obtains

$$
T_{o^{\prime}}^{\prime}=\oint \frac{1}{h^{2}}\left(u_{1}^{\prime 2}+u_{2}^{\prime 2}\right) \mathrm{d} \alpha
$$

If Equation (30) is replaced in Equation (29),

$$
T^{\prime}-T_{o}^{\prime}=\left(x_{1}^{\prime 2}+x_{2}^{\prime 2}\right) m^{\prime}+x_{1}^{\prime} \oint\left(-2 \frac{1}{h^{2}} u_{1}^{\prime} \mathrm{d} \alpha\right)+x_{2}^{\prime} \oint\left(-2 \frac{1}{h^{2}} u_{2}^{\prime} \mathrm{d} \alpha\right)
$$

can be written. Also if Equation (14) is replaced in Equation (31),

$$
\begin{aligned}
T^{\prime}-T_{o}^{\prime}= & \left(x_{1}^{\prime 2}+x_{2}^{\prime 2}\right) m^{\prime}+x_{1}^{\prime}\left(-2 \oint \frac{1}{h^{2}} p_{1}^{\prime} \mathrm{d} \alpha-2 \oint \frac{\mathrm{d} \alpha}{h^{3}(\mathrm{~d} \alpha)^{2}+h(\mathrm{~d} h)^{2}}\left(\mathrm{~d} h \mathrm{~d} u_{1}^{\prime}+h \mathrm{~d} \alpha \mathrm{d} u_{2}^{\prime}\right)\right) \\
& +x_{2}^{\prime}\left(-2 \oint \frac{1}{h^{2}} p_{2}^{\prime} \mathrm{d} \alpha-2 \oint \frac{\mathrm{d} \alpha}{h^{3}(\mathrm{~d} \alpha)^{2}+h(\mathrm{~d} h)^{2}}\left(\mathrm{~d} h \mathrm{~d} u_{2}^{\prime}-h \mathrm{~d} \alpha \mathrm{d} u_{1}^{\prime}\right)\right)
\end{aligned}
$$

is found and by considering Equations (16) and (30) together, we arrive at the relation between the polar moments of inertia and the formula for the area be- 
low:

$$
T^{\prime}-T_{o}^{\prime}=-2\left(F^{\prime}-F_{o^{\prime}}^{\prime}\right)+x_{1}^{\prime}\left(-\oint \frac{1}{h^{2}} \mathrm{~d} u_{2}^{\prime}\right)+x_{2}^{\prime}\left(\oint \frac{1}{h^{2}} \mathrm{~d} u_{1}^{\prime}\right)
$$

\section{Application: The Inverse Motion of the Telescopic Crane}

In the previous sections we emphasized three concepts: geometrical objects as the Steiner point or the Steiner normal, the pole point, and the polar moments of inertia for closed homothetic inverse motions in complex plane. In this section, we want to visualize the experimentally measured motion with these objects. Accordingly, we consider these characteristic directions for this motion.

We will show that how the kinematical objects which used in the previous sections can be applied. Dathe and Gezzi considered human gait in planar motions [5]. As an example, we have chosen the sagittal part of the movement of the telescopic crane at motion. We have chosen the telescopic crane, because the arm of telescopic crane can extend or retract during one parameter closed planar homothetic motion. The motion of telescopic crane has a double hinge and "a double hinge" means that it has two systems, a fixed arm and a moving arm of telescopic crane (Figure 1). There is a control panel of telescopic crane at the origin of fixed system. "L" arm can extend or retract by h parameter.

\subsection{The Mathematical Model by Inverse Motion}

We start by writing the equations of the double hinge in cartesian coordinates. Then we define using the condition $m=0$, the Steiner normal and the total angle in relation to the double hinge.

By taking displacement vector $O O^{\prime}=U$ and $O^{\prime} O=U^{\prime}$, the total angle of rotation $l-k=\alpha$, the motion can be defined by the transformation

$$
X(t)=\frac{1}{h(t)}\left(X^{\prime}-U^{\prime}(t)\right) \mathrm{e}^{-i(\ell(t)-k(t))}
$$

By taking

$$
R(t)=\left(\begin{array}{cc}
\cos (\ell(t)-k(t)) & -\sin (\ell(t)-k(t)) \\
\sin (\ell(t)-k(t)) & \cos (\ell(t)-k(t))
\end{array}\right),
$$

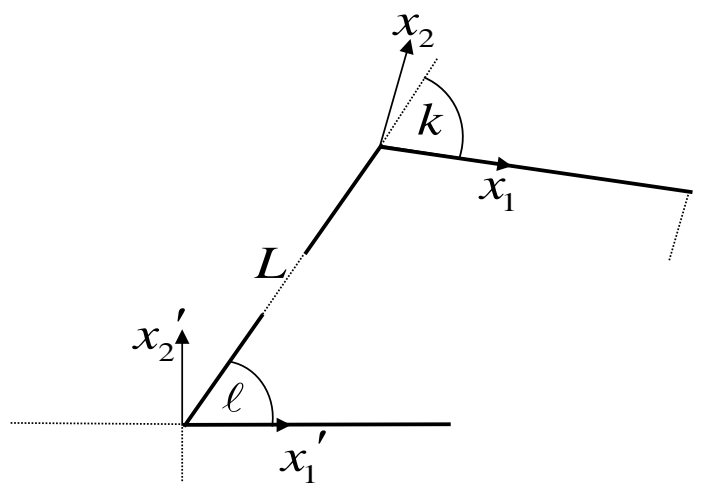

Figure 1. The arms of telescopic crane as a double hinge. 


$$
U(t)=\left(\begin{array}{l}
u_{1}(t) \\
u_{2}(t)
\end{array}\right)=\left(\begin{array}{l}
-L \cos (k(t)) \\
-L \sin (k(t))
\end{array}\right)
$$

we have Equation (5) namely,

$$
X(t)=\frac{1}{h(t)}(R(t))^{\mathrm{T}}\left(X^{\prime}-U^{\prime}(t)\right)
$$

Also we know that $U^{\prime}=-R U$ and

$$
U^{\prime}(t)=\left(\begin{array}{l}
u_{1}^{\prime}(t) \\
u_{2}^{\prime}(t)
\end{array}\right)=\left(\begin{array}{l}
L \cos (\ell(t)) \\
L \sin (\ell(t))
\end{array}\right)
$$

can be written. So the double hinge may be written as

$$
\left.\begin{array}{l}
x_{1}(t)=\frac{1}{h(t)}\left[\cos (\ell(t)-k(t))\left(x_{1}^{\prime}-L \cos (\ell)\right)+\sin (\ell(t)-k(t))\left(x_{2}^{\prime}-L \sin (\ell)\right)\right] \\
x_{2}(t)=\frac{1}{h(t)}\left[-\sin (\ell(t)-k(t))\left(x_{1}^{\prime}-L \cos (\ell)\right)+\cos (\ell(t)-k(t))\left(x_{2}^{\prime}-L \sin (\ell)\right)\right]
\end{array}\right\}
$$

where $\alpha=\ell-k$ is the resulting total angle.

We begin by calculating the time derivative of Equation (37). In this way, we obtain the velocities $\dot{x}_{1}(t), \dot{x}_{2}(t)$ which have to be inserted into Equation (7). Then we have

$$
\begin{aligned}
x_{1} \dot{x}_{2}-x_{2} \dot{x}_{1}= & -\left(x_{1}^{\prime 2}+x_{2}^{\prime 2}\right) \frac{1}{h^{2}}(\dot{\ell}-\dot{k})+x_{1}^{\prime}\left(2 \frac{1}{h^{2}} L \cos \ell(\dot{\ell}-\dot{k})-\frac{1}{h^{2}} \dot{L} \dot{\ell} \cos \ell\right) \\
& +x_{2}^{\prime}\left(2 \frac{1}{h^{2}} L \sin \ell(\dot{\ell}-\dot{k})-\frac{1}{h^{2}} L \dot{\ell} \sin \ell\right)+\frac{1}{h^{2}} L^{2} \dot{k}
\end{aligned}
$$

We now integrate the previous equation using periodic boundary conditions by assuming that the integrands as periodic functions. The periodicity of $f$ implies that integrals of the following types vanish $\oint \mathrm{d} f=\int_{1}^{F} \dot{f} \mathrm{~d} t=\left.f\right|_{1} ^{F}=0$. As a result of this, some of integrals of Equation (38) are not equal to zero. If we use Equation (38) in the area formula

$$
F^{\prime}=\frac{1}{2} \int_{t_{1}}^{t_{2}}\left(x_{1} \dot{x}_{2}-x_{2} \dot{x}_{1}\right) \mathrm{d} t
$$

we finally obtain

$$
\begin{aligned}
2 F^{\prime}= & x_{1}^{\prime} \int_{t_{1}}^{t_{2}}\left(2 \frac{1}{h^{2}} L \cos \ell(\dot{\ell}-\dot{k})-\frac{1}{h^{2}} \dot{L} \dot{\ell} \cos \ell\right) \mathrm{d} t \\
& +x_{2}^{\prime} \int_{t_{1}}^{t_{2}}\left(2 \frac{1}{h^{2}} L \sin \ell(\dot{\ell}-\dot{k})-\frac{1}{h^{2}} \dot{L} \dot{\ell} \sin \ell\right) \mathrm{d} t
\end{aligned}
$$

We can write the integral coefficients in Equation (39) as

$$
\left.\begin{array}{l}
\int_{t_{1}}^{t_{2}}\left(2 \frac{1}{h^{2}} L \cos \ell(\dot{\ell}-\dot{k})-\frac{1}{h^{2}} \dot{L} \dot{\ell} \cos \ell\right) \mathrm{d} t=a^{\prime *} \\
\int_{t_{1}}^{t_{2}}\left(2 \frac{1}{h^{2}} L \sin \ell(\dot{\ell}-\dot{k})-\frac{1}{h^{2}} \dot{L} \dot{\sin \ell}\right) \mathrm{d} t=b^{\prime *}
\end{array}\right\}
$$


Differentiating Equation (36) with respect to $t$ and then replacing both of them in Equation (40), Equation (9) is found for application.

In Section (2.1.1), using Equation (14),

$$
\begin{aligned}
& a^{\prime *}=\underbrace{\int_{t_{1}}^{t_{2}}\left(2 \frac{1}{h^{2}} p_{1}^{\prime} \mathrm{d} \alpha\right)}_{a^{\prime}}+\underbrace{\int_{t_{1}}^{t_{2}}\left(\frac{2 d \alpha}{h^{3}(\mathrm{~d} \alpha)^{2}+h(\mathrm{~d} h)^{2}}\left(\mathrm{~d} h \mathrm{~d} u_{1}^{\prime}+h \mathrm{~d} \alpha \mathrm{d} u_{2}^{\prime}\right)-\frac{1}{h^{2}} \mathrm{~d} u_{2}^{\prime}\right)}_{\mu_{1}^{\prime}} \\
& b^{\prime *}=\underbrace{\int_{t_{2}}^{t_{2}}\left(2 \frac{1}{h^{2}} p_{2}^{\prime} \mathrm{d} \alpha\right)}_{b^{\prime}}+\underbrace{\int_{t_{1}}^{t_{2}}\left(\frac{2 d \alpha}{h^{3}(\mathrm{~d} \alpha)^{2}+h(\mathrm{~d} h)^{2}}\left(\mathrm{~d} h \mathrm{~d} u_{2}^{\prime}-h \mathrm{~d} \alpha \mathrm{d} u_{1}^{\prime}\right)-\frac{1}{h^{2}} \mathrm{~d} u_{1}^{\prime}\right)}_{\mu_{2}^{\prime}}
\end{aligned}
$$

is found and we have a straight line below:

$$
2 F^{\prime}=\left(a^{\prime}+\mu_{1}^{\prime}\right) x_{1}^{\prime}+\left(b^{\prime}+\mu_{2}^{\prime}\right) x_{2}^{\prime} .
$$

In this case, we have the Steiner normal

$$
n^{\prime}=\left(\begin{array}{l}
a^{\prime}+\mu_{1}^{\prime} \\
b^{\prime}+\mu_{2}^{\prime}
\end{array}\right)=L\left(\begin{array}{l}
\int_{t_{1}}^{t_{2}}\left(2 \frac{1}{h^{2}} \cos \ell(\dot{\ell}-\dot{k})-\frac{1}{h^{2}} \dot{\ell} \cos \ell\right) \mathrm{d} t \\
\int_{t_{1}}^{t_{2}}\left(2 \frac{1}{h^{2}} \sin \ell(\dot{\ell}-\dot{k})-\frac{1}{h^{2}} \dot{\ell} \sin \ell\right) \mathrm{d} t
\end{array}\right)
$$

\subsection{The Fixed Pole Point of the Inverse Telescopic Crane Motion}

If Equation (36) is replaced in Equation (26), the pole point $P^{\prime}=\left(\begin{array}{l}p_{1}^{\prime} \\ p_{2}^{\prime}\end{array}\right)$ with the components

$$
\left.\begin{array}{l}
p_{1}^{\prime}=\frac{h}{(\mathrm{~d} h)^{2}+h^{2}(\dot{\ell}-\dot{k})^{2}}(\mathrm{~d} h \dot{\ell} \dot{\ell} \sin \ell-h(\dot{\ell}-\dot{k}) L \dot{\ell} \cos \ell)+L \cos \ell \\
p_{2}^{\prime}=-\frac{h}{(\mathrm{~d} h)^{2}+h^{2}(\dot{\ell}-\dot{k})^{2}}(\mathrm{~d} h \dot{\ell} \cos \ell+h(\dot{\ell}-\dot{k}) L \dot{\ell} \sin \ell)+L \sin \ell
\end{array}\right\}
$$

is found and also by using Equations (43) and (44), we reach at the relation between the Steiner normal and the pole point (Equation (27)).

\subsection{The Polar Moments of Inertia of the Inverse Telescopic Crane Motion}

Using Equations (28) and (37), if Equation (36) is replaced in Equation (29)

$$
T^{\prime}=x_{1}^{\prime} \oint\left(-2 \frac{1}{h^{2}} L(\cos \ell)(\dot{\ell}-\dot{k})\right) \mathrm{d} t+x_{2}^{\prime} \oint\left(-2 \frac{1}{h^{2}} L(\sin \ell)(\dot{\ell}-\dot{k})\right) \mathrm{d} t
$$

is found. By considering Equations (41), (42) and (45) together, we arrive at the relation between the polar moments of inertia and the formula for the area below:

$$
T^{\prime}=-2 F^{\prime}-x_{1}^{\prime} L \oint \frac{1}{h^{2}}(\cos \ell) \dot{\ell} \mathrm{d} t-x_{2}^{\prime} L \oint \frac{1}{h^{2}}(\sin \ell) \dot{\ell} \mathrm{d} t
$$

\section{Acknowledgements}

This study is supported by Ondokuz Mayıs University (Project No. PYO. FEN. 1904. 14.019). 


\section{References}

[1] Steiner, J. (1840) Von dem Krümmungs-Schwerpuncte ebener Curven. Journal für die reine und angewandte Mathematik, 21, 33-63. https://doi.org/10.1515/crll.1840.21.33

[2] Tutar, A. and Kuruoğlu, N. (1999) The Steiner formula and the Holditch theorem for the Homothetic Motions on the Planar Kinematics. Mechanism and Machine Theory, 34, 1-6. https://doi.org/10.1016/S0094-114X(98)00028-7

[3] Müller, H.R. (1978) Verallgemeinerung einer Formel von Steiner. Abhandlungen der Braunschweigischen Wissenschaftlichen Gesellschaft Band, 29, 107-113.

[4] Müller, H.R. (1978) Über Trägheitsmomente bei Steinerscher Massenbelegung. Abhandlungen der Braunschweigischen Wissenschaftlichen Gesellschaft Band, 29, 115-119.

[5] Dathe, H. and Gezzi, R. (2012) Characteristic Directions of Closed Planar Motions. Zeitschrift für Angewandte Mathematik und Mechanik, 92, 2-13.

https://doi.org/10.1002/zamm.201100178

[6] Blaschke, W. and Müller, H.R. (1956) Ebene Kinematik. R. Oldenbourg, München.

[7] Tölke, J. (1978) Steiner-Formeln für die Bahnflächen geschlossener Äquiaffinbewegungen, Sitzungsberı. Österreichische Akademie der Wissenschaften Mathematisch-Naturwissenschaftliche Klasse, 187, 325-337.

[8] Kuruoğlu, N., Düldül, M. and Tutar, A. (2003) Generalization of Steiner Formula for the Homothetic Motions on the Planar Kinematics. Applied Mathematics and Mechanics (English Edition), 24, 945-949. https://doi.org/10.1007/BF02446500

Submit or recommend next manuscript to SCIRP and we will provide best service for you:

Accepting pre-submission inquiries through Email, Facebook, LinkedIn, Twitter, etc. A wide selection of journals (inclusive of 9 subjects, more than 200 journals)

Providing 24-hour high-quality service

User-friendly online submission system

Fair and swift peer-review system

Efficient typesetting and proofreading procedure

Display of the result of downloads and visits, as well as the number of cited articles

Maximum dissemination of your research work

Submit your manuscript at: http://papersubmission.scirp.org/

Or contact jamp@scirp.org 